\title{
CASSON'S INVARIANT AND SURGERY ON KNOTS
}

\author{
by C. D. FROHMAN* and D. D. LONG†
}

(Received 7th May 1990, revised 23rd April 1991)

\begin{abstract}
We show that given a knot in a homology sphere there is a sequence of invariants with the property that if the $n$th invariant does not vanish, then this implies the existence of a family of irreducible representations of the fundamental group of the complement of the knot into $S U(n)$.
\end{abstract}

1980 Mathematics subject classification (1985 Revision) 57N, 20C

\section{Introduction}

The aim of this paper is two-fold. Firstly, we give generalisations of Casson's invariant for a knot $\lambda^{\prime}(K)$, by giving a count of representations of the fundamental group of the knot into the Lie groups $S U(n)$. This seems to be the first such construction of representations into higher dimensional Lie groups. These generalisations have been used by the first named author [3] to show that every fibred knot of genus $g$ has an irreducible representation into $S U(n)$ for some $2 \leqq n \leqq g+1$. In a second direction, we are able to apply the methods to $S U(2)$ representations and by using a larger cycle than Casson, we are able to get slightly more data concerning surgeries than Casson did.

Our main result is that one can extend the definition of Casson's invariant $\lambda^{\prime}(K)$. We summarize this as:

Theorem. Let $K$ be a knot in $S^{3}$. Then there is a sequence of of invariants $\tau_{n, k}$ defined for $k$ and $n$ relatively prime, with the property that $\tau_{n, k} \neq 0$ implies that there is $a$ representation of the fundamental group of the knot into $S U(n)$ sending the longitude to the matrix $e^{(2 \pi i k) / n} I d$.

In fact, we do more than this; we are able to construct whole families of representations mapping the longitude close to the given element. Further, our invariants are defined for knots in any homology sphere by the methods of this paper, however the definition in this case is deferred to [5]. The case that the homology sphere is $S^{3}$ follows from [7], where it is shown that any two free Seifert surfaces for a knot in $S^{3}$ are stably equivalent.

Let $K \subset M^{3}$ be a knot in a homology sphere $M$. We set $X$ to be the exterior of $K$, that is the closure of the complement of a tubular neighbourhood. Let $\lambda, \mu \subset \partial X$ be

- Research supported in part by NSF Grant No. DMS-8701736.

†Research supported in part by NSF Grant No. DMS-8701422. 
choices of a longitude and meridian of $K$, so that $\lambda$ is nullhomologous in $X$ and $\mu$ is nullhomologous in $N(K)$. Dehn surgery on $K$ with slope $q / p$ is the three manifold $M(q / p)$ obtain by gluing a solid torus into $X$ so that the meridian of the solid torus is sent to a curve that is homologous to $p \lambda+q \mu$. The order of $H_{1}(M(q / p))$ is $q$, unless $q=0$, in which case $H_{1}(M(0 / 1))$ is isomorphic to $\mathbf{Z}$.

From the way Casson defines his invariant for knots it is obvious that if $K \subset S^{3}$ is a knot with $\lambda^{\prime}(K) \neq 0$ then for all nonzero integers $n, \pi_{1}(M(1 / n))$ admits an irreducible representation into $S U(2)$. From our viewpoint this is less obvious, but we get the strengthened result:

Theorem 3.1. Let $K$ be a knot in $S^{3}$, such that Casson's invariant of $K$ is nonzero. Then $\pi_{1}\left(S^{3}(q / p)\right)$ admits an irreducible representation into $S U(2)$ for:

(a) all nonzero $q / p$ with absolute values less than or equal to 2;

(b) all $q / p(\neq \infty)$ with absolute value sufficiently large.

Since there has been some interest in this question recently, we observe that $3.1(\mathrm{a})$ implies:

Corollary. Let $K$ be a knot in $S^{3}$, such that Casson's invariant of $K$ is nonzero. Then one cannot obtain $\mathbf{R P}^{3}$ by surgery on $K$.

In contrast it should be noted that the $(-2,3,7)$ pretzel knot has $\lambda^{\prime}(K)=-12$, and $S^{3}(18 / 1)$ has cyclic fundamental group.

Recall that a knot is amphichieral if there is a homeomorphism $h: S^{3} \rightarrow S^{3}$ that sends slopes on $\partial X$ to their negatives. Our methods also yield a weak version of Corollary 4 in [2]:

Theorem 3.2. Let $K \subset S^{3}$ be an amphichieral knot with $\lambda^{\prime}(K) \neq 0$. Then no surgery in $Q-\{0\}$ on $K$ yields a manifold with cyclic fundamental group.

In this paper we shall make no computation of these invariants. If $K$ is a fibred knot our invariants are related to the Lefschetz numbers of certain maps induced on representation varieties by the mondromy action. From this it follows (essentially from [1]), that our $\lambda_{2,1}$ (See §2) is Casson's $\lambda^{\prime}(K)$. This is further exploited in [3] to do some computations; in particular, the Alexander polynomial determines the invariants in this case and it follows from the methods that if $K$ is fibred of genus $g$, then for $2 \leqq n \leqq g+1$ at least one of $\tau_{n, 1}$ is nonzero.

In a somewhat different direction, the first named author and A. Nicas [6] have extended these invariants to the case where $n$ and $k$ are not relatively prime by proving that the analogous cycles in intersection homology can be perturbed in a canonical way to become intersectable.

\section{Special unitary representations}

We begin with some preliminary observations. If $A$ is an $n \times n$ complex matrix, then 
$A^{*}$ will denote the conjugate transpose of $A$. Let $S U(n)$ be the Lie group of all $n \times n$ complex matrices $A$ which have determinant one and satisfy $A^{-1}=A^{*}$. The tangent space at the identity of $S U(n)$ is the space $s u(n)$ of all $n \times n$ traceless complex matrices satisfying $a+a^{*}=0$. If $A \in S U(n)$, then we identify the tangent space at $A$ with $\{\mathbf{x} A \mid \mathbf{x} \in S U(n)\}$, that is to say, we can identify $T_{A}(S U(n))$ with $\operatorname{su}(n)$ using right translation by $A^{-1}$. Further, there is the so-called adjoint representation $\operatorname{Ad:SU}(n) \rightarrow$ $A u t(s u(n))$ which comes from differentiating the map $S U(n) \rightarrow S U(n)$ given by $x \rightarrow g . x . g^{-1}$ at the identity. The kernel of this map is the centre of $S U(n)$.

The matrix exponential allows us to construct paths in $S U(n)$ having any desired tangent vector, so that if $\mathbf{x} \in S u(n)$ and $A \in S U(n)$, then $\gamma(t)=\exp (t \mathbf{x}) . A$ is a path such that $\gamma(0)=A$ and $\gamma^{\prime}(0)=\mathbf{x}$.

Let $\Gamma$ be a finitely generated group; then by $R_{n}(\Gamma)$ we shall mean the set of representations of $\Gamma$ into $S U(n)$ given the structure as a real algebraic set as follows. Choose a generating set $\gamma_{1}, \ldots, \gamma_{s}$ for $\Gamma$. Then $\gamma: R_{n}(\Gamma) \rightarrow S U(n)^{s}$ is given by sending a representation $\rho$ to the point $\left(\rho\left(\gamma_{1}\right), \ldots, \rho\left(\gamma_{s}\right)\right)$. The image of $\gamma$ consists of all those $s$-tuples of matrices in $S U(n)$ which satisfy the relations amongst the $\gamma_{i}$ in $\Gamma$.

The Hilbert basis theorem shows that in fact only finitely many relations suffice to define the image of $\gamma$. If we use another set of generators for $\Gamma$, we obtain an algebraic equivalence between the respective images.

There is an obvious action of $S U(n)$ on $R_{n}(\Gamma)$ conjugation and one sees easily that two irreducible representations $\rho$ and $\rho^{\prime}$ lie in the same orbit for this action if and only if $\operatorname{tr}(\rho(g))=\operatorname{tr}\left(\rho^{\prime}(g)\right)$ for all $g \in \Gamma$. A finite number of traces suffice to describe such orbits and in this way we see that the quotient $R_{n}(\Gamma) / S U(n)=\tilde{R}_{n}(\Gamma)$ is algebraic. Notice that we are working with a genuine quotient space, as opposed to a character variety of representations.

Let $\rho \in R_{n}(\Gamma)$, then a crossed homomorphism $c: \Gamma \rightarrow s u(n)$ (with respect to $\rho$ ) is a map satisfying $c\left(\gamma_{1} \gamma_{2}\right)=c\left(\gamma_{1}\right)+A d_{\rho\left(\gamma_{1}\right)}\left(c\left(\gamma_{2}\right)\right)$. Since $s u(n)$ is a real vector space, the space of crossed homomorphisms is a real vector space. If $\rho_{i}: \Gamma \rightarrow S U(n)$ is a smooth path of representations with $\rho_{0}=\rho$ then

$$
c(\gamma)=\left.\frac{d}{d t} \rho_{t}(\gamma)\right|_{t=0} \rho(\gamma)^{-1}
$$

is a crossed homomorphism.

If $\mathbf{x} \in s u(n)$, then we may form a crossed homomorphism, by setting $c(\gamma)=\mathbf{x}-\boldsymbol{A} d_{\rho(\gamma)}(\mathbf{x})$; such a $c$ we shall call a principal crossed homomorphism. These form a sub-vector space of all crossed homomorphisms. One easily checks that the formula given above applied to the path of representations given by $\rho_{t}(\gamma)=\exp (t \mathbf{x}) \cdot \rho(\gamma) \cdot \exp (-t \mathbf{x})$ gives rise to this crossed homomorphism, and with some work it may be shown that there is a map from tangent vectors to $\tilde{R}_{n}(\Gamma)$ to the quotient space

\{Crossed homomorphisms $\} /\{$ Principal Crossed homomorphisms $\}$.

We denote this latter space by $H^{1}(\Gamma ; a d \rho)$. 
The centre $Z(S U(n))$ is a cyclic group of order $n$, being generated by the matrix $\omega$, which has zeros off the diagonal and $e^{2 \pi i / n}$ on the diagonal. If $\rho: \Gamma \rightarrow S U(n)$ is a representation with $\operatorname{Im}(\rho) \leqq Z(S U(n))$, then principal crossed homomorphisms are all zero and $H^{1}(\Gamma ; a d \rho)=H^{1}(\Gamma) \otimes s u(n)$.

Let $T^{n-1}$ be the torus of diagonal matrices in $S U(n)$. A typical element of $T^{n-1}$ is the diagonal matrix $\left\{\lambda_{1}, \ldots, \lambda_{n}\right\}$ with the product $\Pi \lambda_{i}=1$; we shall take $W$ to be the open subset of this torus consisting of matrices so that no subcollection of the $\lambda_{i}$ 's has product being equal to 1 . For $k$ relatively prime to $n$ we set $W(k)$ to be the connected component of $W$ which contains $\omega^{k}$.

A complete flag for $\mathbf{C}^{n}$ is a sequence of nested subspaces $\{0\}=E_{0} \leqq E_{1} \leqq \cdots \leqq E_{n}=\mathbf{C}^{n}$ so that for each $0 \leqq i \leqq n \operatorname{dim}\left(E_{i} / E_{i-1}\right)=1$. The space of complete flags $F l(n)$ is an $S U(n)$ homogeneous space. The stabiliser of a flag is conjugate to $T^{n-1}$, and hence $F l(n)=$ $S U(n) / T^{n-1}$. Further, each $F l(n)$ is a compact Kahler manifold since it can be realised as a nonsingular projective variety. Observe that $F(1)$ is a point and that for each $n$ there is a fibre bundle $F l(n-1) \rightarrow F l(n) \rightarrow \mathbf{C P}(n-1)$ where the projection is given by sending the flag $E_{0} \leqq E_{1} \leqq \cdots \leqq E_{n}$ to $E_{1}$. It follows that $F l(2)=\mathbf{C} P(1)$. From our point of view we need only:

Lemma 1.1. The Euler characteristic of $F l(n)$ is nonzero.

Proof. The fibre bundles $F l(n-1) \rightarrow F l(n) \rightarrow \mathbf{C P}(n-1)$ can easily be seen to be holomorphic. It follows that the Deligne criterion for the degeneration of a spectral sequence can be applied to show that the sequence associated with the above fibration collapses at the $E^{2}$ term. Since $\mathbf{C P}(n-1)$ is simply connected the monodromy of the bundle is trivial, so that $H^{*}(F l(n))=H^{*}(F l(n-1)) \otimes H^{*}(\mathrm{C} P(n-1))$.

Therefore the Betti numbers of $F l(n)$ are zero in odd dimensions and nonzero in all even dimensions less than or equal to $n^{2}-n$. Whence the Euler characteristic of $F l(n)$ is nonzero, completing the proof.

There is an action of $T^{n-1}$ on $R_{n}(\Gamma)$ by conjugation; we shall denote the quotient under this action by $\hat{R}_{n}(\Gamma)$. There is an obvious map $\hat{R}_{n}(\Gamma) \rightarrow \widetilde{R}_{n}(\Gamma)$. If $\rho$ is an irreducible representation then the inverse image of $[\rho] \in \tilde{R}_{n}(\Gamma)$ in $\hat{R}_{n}(\Gamma)$ is the flag manifold $F l(n)$.

Let $F$ be a compact orientable surface with one boundary component. A standard set of generators for $\pi_{1}(F)$ consists of a family of simple closed curves sharing a common basepoint on $\partial F$ and otherwise disjoint, so that the loop $\prod_{i=1}^{g}\left[a_{i}, b_{i}\right]$, (where $\left[a_{i}, b_{i}\right]$ denotes the commutator $\left.a_{i} b_{i} a_{i}^{-1} b_{i}^{-1}\right)$ corresponds to the conjugacy class of $\partial F$ in $\pi_{1}(F)$. Corresponding to such a choice of curves we see that $R_{n}\left(\pi_{1}(F)\right)=S U(n)^{2 g}$. From this it is easy to deduce that $T_{\rho}\left(R_{n}\left(\pi_{1}(F)\right)\right.$ is the same as the space of crossed homomorphisms. Define a map

$$
\partial: R_{n}\left(\pi_{1}(F)\right) \rightarrow S U(n)
$$

to be given in coordinates by $\partial(\rho)=\rho\left(\prod_{i=1}^{g}\left[a_{i}, b_{i}\right]\right)$. Then we can identify the tangent 
space of $S U(n)^{2 \theta}$ at $\left(A_{1}, \ldots, A_{g}, B_{1}, \ldots, B_{g}\right)$ with $s u(n)^{2 g}$ by right translation. With this identification it is easy to check from the co-ordinate formula for

$$
D \partial: T_{\left(A_{1}, \ldots, A_{g}, B_{1}, \ldots, B_{g}\right)}\left(S U(n)^{2 g}\right) \rightarrow \operatorname{su}(n)
$$

that:

Lemma 1.2 (see [1]). The map $\partial$ is a submersion at $\left(A_{1}, \ldots, A_{g}, B_{1}, \ldots, B_{g}\right)$ if and only if the representation $\left(A_{1}, \ldots, A_{g}, B_{1}, \ldots, B_{g}\right)$ is irreducible. Hence the point $d \in T^{n-1}$ is $a$ regular value of $\partial$ if and only if $d \in W$.

Let $M^{3}$ be a closed oriented 3-manifold that is a homology sphere. By definition, a $k$ not $K \subset M$ is an oriented simple closed curve in $M$. A free Seifert surface for $K$ is an embedded 2-sided surface $F$ in $M$ with $\partial F=K$, with the further property that if $H_{1}=F \times I$ is a collaring of $F$, then $H_{2}=C l\left(M \backslash H_{1}\right)$ is a handlebody. It is well known [1] that every knot has a free Seifert surface. In the notation established above we will identify $\pi_{1}(F)$ and $\pi_{1}(F \times l)$ via inclusion. Set $S=\partial H_{1}$. Then we may see $S$ as the union of two surfaces $F_{0}$ and $F_{1}$ so that $F_{0} \cap F_{1}=K$. Giving $H_{1}$ the orientation inherited from $M$ and $S$ the orientation qua $\partial H_{1}$, then $F_{0}$ is a subsurface of $S$ and this gives an orientation to $K$.

With generators $a_{i}$ and $b_{i}$ for $\pi_{1}(F)$ as above, for $j=0,1$, we may choose bases $a(j)_{i}$ and $b(j)_{i}$ so that under the inclusion $F_{j} \rightarrow F \times I$ the elements $a(j)_{i} \rightarrow a_{i}$ etc. This gives a presentation for $\pi_{1}(S)$ with the $4 g$ generators $\left\{a(j)_{i}, b(j)_{i}\right\}$ and relator $\Pi\left[a(0)_{i}, b(0)_{i}\right]=$ $\Pi\left[a(1)_{i}, b(1)_{i}\right]$. By hypothesis, the handlebodies $H_{1}$ and $H_{2}$ form a Heegaard splitting for $M$. Let $\tau_{i}: H_{i} \rightarrow H$ be orientation preserving homeomorphisms of the $H_{i}$ with some fixed handlebody $H$. We define $\phi: S \rightarrow S$ by restricting $\tau_{2} \tau_{1}^{-1}$ to a map $\partial H_{1} \rightarrow \partial H_{2}=S$. Using this map we obtain a presentation for $\pi_{1}(M)$ :

$$
\pi_{1}(M)=\left\langle a(j)_{i}, b(j)_{i} \mid a(0)_{i}=a(1)_{i}, b(0)_{i}=b(1)_{i}, \phi_{*} a(0)_{i}=\phi_{*} a(1)_{i}, \phi_{*} b(0)_{i}=\phi_{*} b(1)_{i}\right\rangle .
$$

We may similarly obtain a presentation for $\pi_{1}(M \backslash K)$. For $j=0,1$ let $u(j)_{i}$ and $v(j)_{i}$ be the words obtained from $\phi_{*} a(j)_{i}$ and $\phi_{*} b(j)_{i}$ by replacing every occurrence of $a(1)_{i}$ and $b(1)_{i}$ by $\mu^{-1} \cdot a(1)_{i} \cdot \mu$ and $\mu^{-1} \cdot b(1)_{i} \cdot \mu$. Then:

$$
\begin{aligned}
& \pi_{1}(M \backslash K)= \\
& \quad\left\langle a(j)_{i}, b(j)_{i}, \mu \mid\left[\mu, \Pi\left[a(0)_{i}, b(0)_{i}\right]\right], a(0)_{i}=a(1)_{i}, b(0)_{i}=b(1)_{i}, u(0)_{i}=u(1)_{i} v(0)_{i}=v(1)_{i}\right\rangle .
\end{aligned}
$$

By slight abuse of notation we will denote the representation spaces of $\pi_{1}\left(F_{0}\right), \pi_{1}\left(F_{1}\right)$ and $\pi_{1}(S)$ by $R_{n}\left(F_{0}\right)$, etc. For $i=1,2$ we have maps $\partial_{i}: R_{n}\left(F_{i}\right) \rightarrow S U(n)$ analogous to $\partial$.

If $\rho \in R_{n}(S)$, then using the fact that $S=F_{0} \cup F_{1}$ we may obtain $\rho_{0}: \pi_{1}\left(F_{0}\right) \rightarrow S U(n)$ and $\rho_{1}: \pi_{1}\left(F_{1}\right) \rightarrow S U(n)$, so that we may identify $R_{n}(S)$ with:

$$
\left\{\left(\rho_{0}, \rho_{1}\right) \in R_{n}\left(F_{0}\right) \times R_{n}\left(F_{1}\right) \mid \partial_{0} \rho_{0}=\partial_{1} \rho_{1}\right\}
$$

By the diagonal of $R_{n}(S)$ we shall mean the set $P_{1}$ of representations $\left(\rho_{0}, \rho_{1}\right) \in R_{n}(S)$ 
with the property that $\rho_{0}\left(a(0)_{i}\right)=\rho_{1}\left(a(1)_{i}\right)$ and $\rho_{0}\left(b(0)_{i}\right)=\rho_{1}\left(b(1)_{i}\right)$. Further, this identification allows us to define in an obvious way a map $\partial_{0}: R_{n}(S) \rightarrow S U(n)$.

If $A$ is a subset of $S U(n)$, then we shall use a subscript $A$ to denote the inverse image of $A$ under $\partial_{0}$; so that for example $R_{A, n}(S)=\partial_{0}^{-1}(A)$. Observe that since the sets $\omega$ and $W$ consists of regular values of $\partial_{0}$, the sets $R_{\omega, n}(S)$ and $R_{W, n}(S)$ are smooth manifolds. If $n$ and $k$ are relatively prime, we write $R_{k, n}(S)$ for the inverse image of $\omega^{k}$.

Lemma 1.3. Suppose that $n$ and $k$ are relatively prime. Then:

(a) The map $\partial_{0}: R_{W(k), n}(S) \rightarrow W(k)$ is a fibre bundle map, with fibre $R_{k, n}(S)$.

(b) The map $\partial_{0}: \hat{R}_{W(k), n}(S) \rightarrow W(k)$ is a bundle map with fibre $\hat{R}_{k, n}(S)$.

(c) The natural map $\hat{R}_{k, n}(S) \rightarrow \widetilde{R}_{k, n}(S)$ is a bundle map with fibre the complex flag manifold $F l(n)$.

Proof. The first two parts follow from the Ehresmann fibration theorem and the fact that $\partial_{0}$ is a submersion onto $W(k)$. The third part we have observed earlier.

Observe that $P_{1}$ corresponds to those representations of $R_{n}(S)$ that are restrictions of representation of $\pi_{1}\left(H_{1}\right)$. Further, setting $P_{2}=\phi\left(P_{1}\right)$, this consists of representations of $\pi_{1}(S)$ that are restrictions of representations of $\pi_{1}\left(H_{2}\right)$.

Clearly $P_{1}$ and $P_{2}$ are homeomorphic to $S U(n)^{2 g}$. Also, the points of $P_{1} \cap P_{2}$ are in one-one correspondence with representations of $\pi_{1}(M)$ into $S U(n)$.

A representation is said to be projectively trivial if its image lies in the centre of $S U(n)$. The intersection of $P_{1}$ and $P_{2}$ at such a representation is easily analysed.

Proposition 1.4. The intersection of $P_{1}$ and $P_{2}$ at a projectively trivial representation is an isolated point of intersection.

Proof. The tangent space of $R_{n}(S)$ at a projectively trivial representation is $H^{1}(S) \otimes s u(n)$. The equations for the tangent spaces of $P_{1}$ and $P_{2}$ at that point give a presentation for $H^{1}(M) \otimes s u(n)=0$. It follows that $P_{1}$ and $P_{2}$ are transverse at any projectively trivial representation.

We need two maps that are derived from the fact that $R_{n}(S) \subseteq R_{n}\left(F_{0}\right) \times R_{n}\left(F_{1}\right)$. By projection, for each $k$ we have:

$$
\tilde{\theta}_{k}: \widetilde{R}_{k, n}(S) \rightarrow \tilde{R}_{k, n}\left(F_{0}\right) \times \tilde{R}_{k, n}\left(F_{1}\right)
$$

Also for any $A$ in $W$ we have:

$$
\hat{\theta}_{A}: \hat{R}_{A, n}(S) \rightarrow \hat{R}_{A, n}\left(F_{\mathrm{o}}\right) \times \hat{R}_{A, n}\left(F_{1}\right)
$$

For the sake of notational simplicity, we refer to $\tilde{R}_{k, n}\left(F_{0}\right) \times \tilde{R}_{k, n}\left(F_{1}\right)$ as $\tilde{R}_{k}$ and $\hat{R}_{A, n}\left(F_{0}\right) \times \hat{R}_{A, n}\left(F_{1}\right)$ as $\hat{R}_{A}$. 
Observe that the projection $r: \hat{R}_{k} \rightarrow \tilde{R}_{k}$ is fibre bundle with fibre $F l(n) \times F l(n)$. Let $\hat{Q}_{1}$ and $\hat{Q}_{2}$ be those subsets of $\hat{R}_{W}$ obtained by projecting $P_{1} \cap R_{W, n}(S)$ and $P_{2} \cap R_{W, n}(S)$ down into $\hat{R}_{W}$ using $\hat{\theta}_{W}$.

Proposition 1.5. An $S U(n)$ representation of $\pi_{1}(M \backslash K)$ can be constructed for each point of intersection of $\hat{Q}_{1}$ and $\hat{Q}_{2}$.

Every representation of $\pi_{1}(M \backslash K)$ with the property that the longitude of $K$ is sent to an element of $W$ arises from an intersection of $\hat{Q}_{1}$ and $\hat{Q}_{2}$.

Proof. For the first part, we use the presentation of $\pi_{1}(M \backslash K)$ given above. Given a point of intersection of $\hat{Q}_{1}$ and $\hat{Q}_{2}$, there are points of $P_{1}$ and $P_{2} ;$ (write these as $(\rho, \rho)$ and $(\xi, \xi))$ and an element $\mu$ of $T^{n-1}$ so that:

$$
(\rho, \rho)=\left(\mu^{-1} \cdot \xi \cdot \mu, \mu^{-1} \cdot \xi \cdot \mu\right)
$$

The representations $\rho$ and $\xi$ give representations of $\pi_{1}\left(H_{1}\right)$ and $\pi_{1}\left(H_{2}\right)$, $\mu$ gives the value of the meridian. Equation (2) shows that the relators of $\pi_{1}(M \backslash K)$ are satisfied.

The second part is the reverse observation, after performing a preliminary conjugacy, so that $\mu \in T^{n-1}$.

We also observe the following proposition; for this, we need to restrict to knots in a simply connected manifold.

Proposition 1.6. Suppose that $\gamma(s)$ is a sequence of representations in $\hat{Q}_{1} \cap \hat{Q}_{2}$ so that the image of the longitude under the representation corresponding to $\gamma(s)$ is the diagonal matrix $d(s)$. Suppose that $d(s) \rightarrow I$ as $s \rightarrow \infty$.

Then there is a representation $\rho_{0}$ that is the limit of a subsequence of the representations $\gamma(s)$ as $s \rightarrow \infty$, and this representation is not projectively trivial.

Proof. By compactness, there is obviously a subsequence which converges in the compact open topology. Relabel, so that this is the whole sequence. We need to show that the limit of this sequence is not projectively trivial. We shall do this by constructing from our sequence another representation (not necessarily into $S U(n)$ ) where the image of the meridian is in the centre, but some element of the commutator subgroup is nontrivial. Since for a knot in a simply connected 3-manifold the normal closure of the meridian generates the knot group, this will be a contradiction.

Suppose that $G$ is the knot group, and we have chosen a generating set $\mu, \alpha_{1}, \ldots, \alpha_{k}$, where $\mu$ is a meridian (in particular, $\mu$ generates the abelianisation of $G$ ) and the $\alpha_{i}$ lie in the commutator subgroup. By conjugacies which we may suppose to converge, we can suppose that $\gamma(s)(\mu)$ is a diagonal matrix. Consider the last column of $\gamma(s)\left(\alpha_{i}\right)$. It cannot be that, for every $i$, this column only contains a single nonzero entry, namely the diagonal, since this would make the representation reducible; contrary to hypothesis. So by subsequence and renumbering, we may suppose without loss that $\gamma(s)\left(\alpha_{1}\right)$ always has its $(1, n)$ entry nonzero. By further subsequence, and again without loss, we may suppose 
further that $\left|\gamma(s)\left(\alpha_{1}\right)_{1, n}\right| \geqq\left|\gamma(s)\left(\alpha_{i}\right)_{j, n}\right|$ for all $1 \leqq i \leqq k$ and $1 \leqq j \leqq n-1$. By further subsequence, we may arrange that for each $\alpha_{i}$ all the limits as $s \rightarrow \infty$ $\left\{\gamma(s)\left(\alpha_{i}\right)_{j, n} / \gamma(s)\left(\alpha_{1}\right)_{1, n}\right\}_{1 \leqq j \leqq n-1}$ exist.

We now define a map $R$ from $G^{\prime}$ to column vectors which are $(n-1) \times 1$ as follows. If $\alpha \in G^{\prime}$, set $R(\alpha)$ to be the limit as $s \rightarrow \infty$ of the column vector, $\left\{\gamma(s)(\alpha)_{i, n} / \gamma(s)\left(\alpha_{1}\right)_{1, n}\right\}$ $1 \leqq j \leqq n-1$; providing this limit exists. By hypothesis, this limit exists on the $\alpha_{i}^{\prime} s$. From this it follows easily that $R$ exists on $\mu^{q} \cdot \alpha_{i} \cdot \mu^{-q}$ for each $q$. Inductively, if $\alpha$ and $\beta$ are elements in $G^{\prime}$ for which $R$ is defined, then $R(\alpha \cdot \beta)$ is defined and is $R(\alpha)+R(\beta)$. The fact that $R$ is well defined follows from the invariant definition given above. Notice that $R$ is not identically zero, since it has a 1 in the $(1, n)$ entry for $\alpha_{1}$.

If now the subsequence $\gamma(s)$ converged to a projectively trivial representation, we would have that $\mu$ would converge to the diagonal matrix with say $e^{(2 \pi i k) / n}$ on the diagonal, and one can easily check that

$$
\sigma: \mu^{q} \cdot \alpha \rightarrow\left(e^{(2 \pi i k) / n} I d\right)^{q} \cdot\left[\frac{\text { Identity } \mid R(\alpha)}{R(\alpha)^{*} \mid 1}\right]
$$

is a representation of the required type.

Remark. This is the $P U(n)$ isolation of the trivial representation; see Proposition 3.4 in [8].

\section{The invariants $\tau_{n, k}$}

In this section we define two families of invariants of an oriented knot in an oriented homology sphere with free Seifert surface. In fact these invariants are independent of the surface chosen, but we will not address that issue here. In the case that the homology sphere is $S^{3}$, this will follow from [7] where stable equivalence of free Seifert surfaces is proven. Moreover, in the particular case that the knot is fibred, this becomes a triviality in all cases.

After defining them we shall show that the two invariants are related. We then use the Ehresmann fibration theorem to show that the nonvanishing of these invariants implies that the group of the knot has a large family of $S U(n)$ representations.

In order to begin, we need to orient everything. Let $R^{i}(S)$ be the space of irreducible representations of $S$. Orient $S$ as the boundary of a collaring of the free Seifert surface and choose a standard generating system $\left\{a_{i}, b_{i}\right\}$ so that $a_{i}$ meets $b_{i}$ in one point. Let the map $\partial_{0}$ be the boundary map of $F_{0}$ and orient $R^{i}(S)$ using the inverse image orientation and $\tilde{R}^{i}(S)=R^{i}(S) / S U(n)$ the quotient orientation. Orient $R_{k, n}(S)$ as the inverse image of $\omega^{k}$ under $\partial_{0}$ and $\tilde{R}_{k, n}(S)=R_{k, n}(S) / S U(n)$ as quotient. Orient $P_{1}$ and use this to orient $P_{2}$ qua $\phi^{\# \prime}\left(P_{1}\right)$. Orient $\widetilde{P}_{1}$ and $\widetilde{P}_{2}$ as quotients. Make $\tilde{P}_{i}$ transverse to $\tilde{R}_{k, n}(S)$ and let $\tilde{S}_{1}$ and $\widetilde{S}_{2}$ be their oriented intersections. Set $s_{i}: \widetilde{S}_{i} \rightarrow \widetilde{R}_{k, n}(S)$ to be the inclusions.

Finally we consider maps $\tilde{\theta}_{k} s_{i}: \widetilde{S}_{i} \rightarrow \tilde{R}_{k}(S)$, where $\tilde{\theta}_{k}$ is the map defined after Proposition 1.4. We set $\tau_{n, k}$ to be the oriented intersection number 


$$
\tau_{n, k}=\left[\tilde{\theta}_{k} s_{1}\right] \cdot\left[\tilde{\theta}_{k} s_{2}\right] .
$$

Setting $d_{2}$ to be the intersection number of $Q_{1}$ and $Q_{2}$ in $S U(n)^{2 g}$ we define:

$$
\begin{aligned}
\lambda_{n, k} & =(-1)^{g} \tau_{n, k} / d_{2} & & \text { if } n \text { is even } \\
& =\tau_{n, k} & & \text { if } n \text { is odd. }
\end{aligned}
$$

Casson's invariant is $\lambda_{2,1}$ (See [3], Chapter V]). In the case that $K$ is a fibred knot then one has that $\lambda_{n, k}$ is the Lefschetz number of the monodromy acting on $\tilde{R}_{k, n}(S)$.

In order to see that the numbers $\lambda_{n, k}$ are independent of the perturbations involved, it is useful to give a cohomological interpretation of these invariants.

$$
\tilde{R}^{i}(S) \stackrel{\text { inclusion }}{\longleftarrow} \tilde{R}_{k, n}(S) \rightarrow \tilde{R}_{k} .
$$

Let $p$, be the pushforward

$$
p_{!}: H^{*}\left(\tilde{R}_{k, n}(S)\right) \rightarrow H^{*-n^{2}+1}\left(\tilde{R}_{k}\right)
$$

(This is given by Poincare duality on $\widetilde{R}_{k, n}(S)$, the induced mapping on homology, followed by another application of duality.) If we view $\tilde{Q}_{1}$ and $\tilde{Q}_{2}$ as locally finite cycles, then they have duals $q_{1}$ and $q_{2}$ and we see that $\tau_{n, k}$ is given by

$$
\tau_{n, k}=p_{!} i^{*} q_{1} \cup p_{i} i^{*} q_{2}\left[\widetilde{R}_{k}\right]
$$

That the invariants $\tau_{n, k}$ are well defined for knots in $S^{3}$ follows from [7], where it is shown that any two free Seifert surfaces are stably equivalent, and applying the analysis along the lines of [1]. The general case will be dealt with in [5].

It follows from our analysis of representations of $\pi_{1}(M \backslash K)$ that if $\lambda_{n, k} \neq 0$ then there exist representations of $\pi_{1}(M \backslash K)$. We now strengthen this result to show that there is a family of representations.

This is done by defining an associated intersection number. Let $\hat{P}_{i}$ be the quotient of $P_{i}$ by conjugation by $T^{n-1}$. Notice that at least at an irreducible point of $\hat{P}_{i}$ the map $\hat{P}_{i} \rightarrow \widetilde{P}_{i}$ is a fibre bundle with fibre $F l(n)$. If we perturb $P_{i}$ so that it is transverse to $\tilde{R}_{k, n}(S)$ then we may lift the perturbation so that $\hat{P}_{i}$ is made transverse to $\hat{R}_{k, n}(S)$ inside $\hat{R}^{i}(S)$. Let $\hat{S}_{i}$ be the intersection of $\hat{P}_{i}$ with $\hat{R}_{k, n}(S)$. Let $\hat{S}_{i}: \hat{S}_{i} \rightarrow \hat{R}_{k, n}(S)$ be the inclusions. Define $\sigma_{n, k}$ to be the intersection number of $\hat{\theta}_{k} \hat{s}_{1}$ with $\hat{\theta}_{k} \hat{s}_{2}$ in $\hat{R}_{k}$. Then we have:

Proposition 2.1. If $\lambda_{n, k} \neq 0$, then $\sigma_{n, k} \neq 0$.

Proof. We need the following lemma:

Lemma 2.2. Let $F \rightarrow E \rightarrow B$ be a fibre bundle where all the spaces are orientable closed manifolds. Assume that these hypotheses also hold for $F_{i} \rightarrow E_{i} \rightarrow B_{i} i=1,2$. Suppose that there are maps making the following diagram commute. 


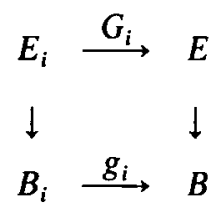

and that $\operatorname{dim}\left(F_{1}\right)+\operatorname{dim}\left(F_{2}\right)=\operatorname{dim}(F)$ and $\operatorname{dim}\left(B_{1}\right)+\operatorname{dim}\left(B_{2}\right)=\operatorname{dim}(B)$. Then after orienting the objects involved, the intersection number of $G_{1}$ and $G_{2}$ (up to sign) is the product of the intersection number of $g_{1}$ and $g_{2}$ in $B$ and of $F_{1}$ and $F_{2}$ in some fibre $F$.

Since $\hat{S}_{i} \rightarrow \tilde{S}_{i}$ is a fibre bundle with fibre $F l(n)$ and since in a fibre $F l(n) \times F l(n)$ of $\hat{R}_{k} \rightarrow \widetilde{R}_{k}$ the fibre of $\hat{S}_{i} \rightarrow \widetilde{S}_{i}$ corresponds to the diagonal in $F l(n) \times F l(n)$, we have that up to sign $\sigma_{n, k}$ is the product of the Euler characteristic of $F l(n)$ (which is nonzero by Lemma 1.1) with $\lambda_{n, k}$. The proposition follows.

Theorem 2.3. Suppose that $K$ is a knot in $M$ with free Seifert surface $F$ and suppose that the corresponding $\lambda_{n, k}$ is nonzero. Then for each $l \in W(k)$ there exists an irreducible representation of $\pi_{1}(M \backslash K)$ so that the longitude of $K$ is sent to l.

Proof. The map $\partial: \hat{R}_{W(k)}(S) \rightarrow W(k)$ is a bundle map with fibre $\hat{R}_{k}(S)$. Hence in the fibre over $l$ the sets $\hat{P}_{1} \cap \hat{R}_{l, n}$ and $\hat{P}_{2} \cap \hat{R}_{l, n}$ must intersect, otherwise we could make $\hat{P}_{1}$ and $\hat{P}_{2}$ transverse to $\hat{R}_{l, n}$ and exhibit $\hat{S}_{l, 1}$ and $\hat{S}_{2, l}$ as disjoint after projection into $\hat{R}_{l, n}\left(F_{0}\right) \times \hat{R}_{l, n}\left(F_{1}\right)$. Since $W(k)$ is contractible and $\partial$ is a bundle map, this implies that $\sigma_{n, k}=0$, which by Proposition 2.1 implies $\lambda_{n, k}=0$, contradicting the hypothesis.

Observe that the last theorem implies that if $\lambda_{n, k}$ is nonzero then the knot group has a family of conjugacy classes of irreducible $S U(n)$ representations of real dimension at least $n-1$.

\section{Surgery on a knot in a simply connected manifold}

In this section we use results that were derived in the previous two sections to prove the existence of irreducible $S U(2)$ representations of the fundamental group of the result of many surgeries on a knot with nonzero Casson's invariant in a simply connected manifold.

Let $T^{2}=S^{1} \times S^{1}$, where we use the complex numbers of norm one as a model for the circle. The fundamental group of $T^{2}$ is $\mathbf{Z} \oplus \mathbf{Z}$. Let $\mu$ and $\lambda$ be generators of $\pi_{1}\left(T^{2}\right)$. If $\rho: \pi_{1}\left(T^{2}\right) \rightarrow S U(2)$ then $\rho$ is completely determined by $\rho(\mu)$ and $\rho(\lambda)$. Since $\pi_{1}\left(T^{2}\right)$ is abelian the matrices $\rho(\mu)$ and $\rho(\lambda)$ can be simultaneously diagonalised. On the other hand if $M$ and $L$ are a pair of matrices that can be simultaneously diagonalised then they define a representation of $\pi_{1}\left(T^{2}\right)$ by giving values of the representation for $\mu$ and $\lambda$. Further, if a pair of matrices $(L, M)$ in $S U(2)$ can be simultaneously diagonalised then they are simultaneously conjugate to $\left(L^{-1}, M^{-1}\right)$. The diagonal matrices in $S U(2)$ are homeomorphic to $S^{1}$ by the mapping that sends each matrix to its upper left hand 


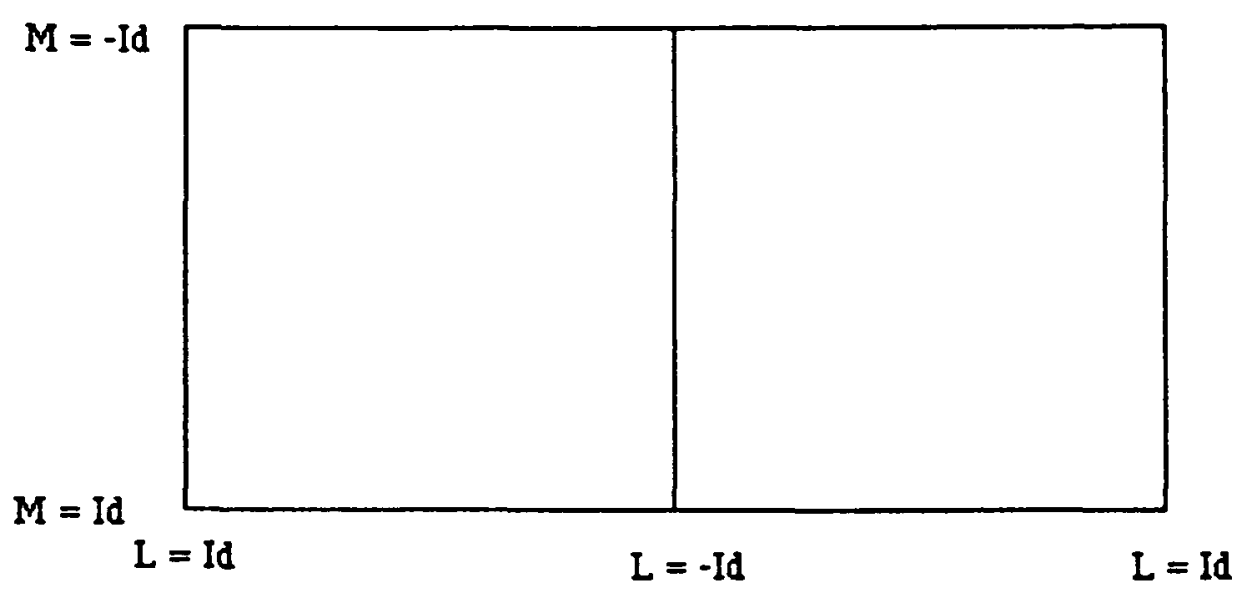

FIGURE 1

entry. Hence the space of pairs of diagonal matrices is homeomorphic to $S^{1} \times S^{1}$. Let $t: S^{1} \times S^{1} \rightarrow S^{1} \times S^{1}$ be the involution given by taking the complex conjugate of both entries. (This is a description of the so-called hyperelliptic involution.) One has easily that the space of pairs of diagonal matrices up to conjugacy is homeomorphic to the quotient of $S^{1} \times S^{1}$ by the hyperelliptic involution.

This quotient space is commonly called the pillow case, and we will denote it by $P$. The space $P$ is topologically a two-sphere and it has the geometric structure of a Euclidian orbifold with 4 singular points with cone angle $\pi$.

We depict $P$ in Fig. 1; it should be imagined as two squares glued together along their boundaries. One square is the front and one square is the back. The bottom seam and the top seam are the loci $M=I d$ and $M=-I d$. The left seam and the right seam are the loci $L=I d$ and $L=-I d$. The arc joining $(I d, I d)$ to $(-I d,-I d)$ on the front is the locus $L=M$. The arc on the back joining $(I d, I d)$ to $(-I d,-I d)$ is the locus $L=M^{-1}$. The loci $M^{q} L^{p}=I d$ are geodesics in the Euclidian structure that pass through the point $(I d, I d)$. For our purposes, it is best to write this equation as $M^{q}=L^{-p}$. From this it is clear that the slope of the geodesic in $P$ is $-p / q$.

If $|-p / q|>1 / 2$ then following the geodesic out from $(I d, I d)$ it will cross the locus $M=-I d$ before it touches the locus $L=I d$ again. In the critical case $|-p / q|=1 / 2$ the first time the geodesic touches the locus $L=I d$ again is at the point $(I d,-I d)$. This is indicated in the second figure below.

Theorem 3.1. Let $K$ be a knot in a simply connected three-manifold $M$, such that Casson's invariant of the knot is nonzero. The result of surgery on the knot $M(q / p)$ admits an irreducible representation into $S U(2)$ for:

(a) all nonzero $q / p$ with absolute value less than or equal to 2 ,

(b) all $q / p(\neq \infty)$ with absolute value sufficiently large. 


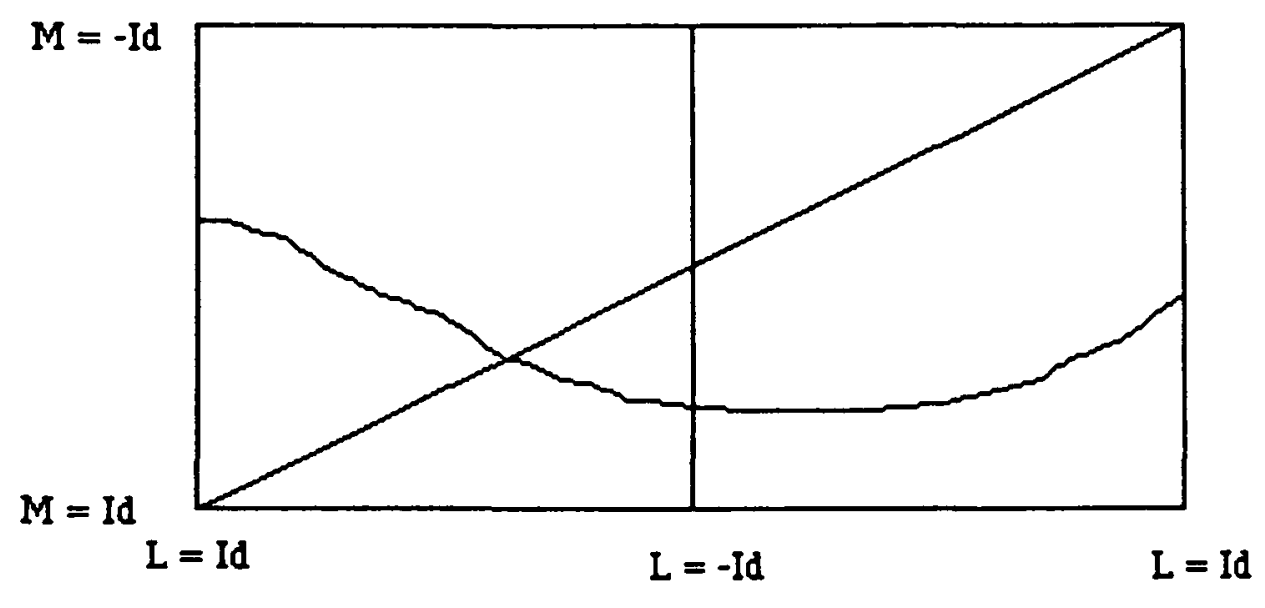

FIGURE 2

Proof. We will denote the space of conjugacy classes of $S U(2)$ representations of the fundamental group $C l(M \backslash N(K))$ by $R(K)$. There is a map $b: R(K) \rightarrow P$ given by restricting representations to the fundamental group of the boundary torus. Since we are working up to conjugacy this map is independent of choice of basepoint, and choice of the representative of the peripheral subgroup. We coordinatize $P$ so that $L$ corresponds to the longitude to the knot and $M$ corresponds to the meridian.

Next since $K$ is a knot in a simply connected manifold, Proposition 1.4 implies that the closure of the image of the irreducible representations of the fundamental group of $K$ misses the loci $M=I d$ and $M=-I d$. If now $\lambda_{2,1}(K) \neq 0$ then by Theorem 2.3 we can find an arc in the image of the irreducible representations of the fundamental group of $K$ whose endpoints lie on $L=I d$ that is parameterised by the value of the longitude away from the locus $L=I d$. Notice that by Proposition 1.6 such an arc cannot have endpoints in the loci $M= \pm I d$.

Such an arc is shown in Fig. 2. From our observations about the loci $M^{q} L^{p}=I d$ we see that this arc must intersect all such loci with $|-p / q| \geqq 1 / 2$. This shows that every surgery on the knot of slope $|q / p| \leqq 2$ admits an irreducible $S U(2)$ representation. Moreover, it is clear from the picture that there is an $\varepsilon>0$ (where the $\varepsilon$ depends on the knot) so that if $|-p / q|<\varepsilon$ then the locus $M^{q} L^{p}=I d$ intersects the image of the irreducible representations. The theorem follows.

Suppose now that $K$ is a knot in a simply connected manifold and there exists a homeomorphism of the complement of $K$ whose restriction to the boundary sends slopes to their negative. This is true if for instance $K$ is an amphichieral knot in $S^{3}$. Suppose then that $q / p$ is any slope other than zero or infinity.

Notice that the geodesics through the trivial representation in the pillow case, that have slope $p / q$ or $-p / q$ partition the pillow case into diamond shaped regions. The diamonds intersect the locus $L=I d$ in vertices. Suppose that $\lambda^{\prime}(K) \neq 0$. As above there 
exists an arc in the closure of the image of the irreducible representations of the knot group that runs monotonically around the pillow case with its endpoints on the locus $L=I d$. The interior points of the arc correspond to irreducible representations of the knot group. The endpoints may correspond to either irreducible representations or abelian representations of the knot group. The arc must intersect the boundary of one of the diamond shaped regions. If this arc intersects the boundary of a diamond away from the locus $L=I d$ then the fundamental group arising as a result of surgery on $K$ admits an irreducible representation into $S U(2)$. If the closure of the image of the arcs of representations intersect the boundary of the diamond in one of the points where the longitude is sent into the identity then either there exists an irreducible representation into $S U(2)$ or there is an abelian representation at that point. Using a subconvergence argument we can produce a nonzero tangent vector corresponding this arc of representations. As in [4] this tangent vector corresponds to an irreducible representation of the fundamental group of the knot into the congruence transformations of the Euclidian plane. In any case the fundamental group of the result of surgery on the knot is not cyclic. (Actually, we may alter a few words in Proposition 1.6 and deduce the same result). We have proved:

Theorem 3.2. Let $K \subset S^{3}$ be an amphichieral knot with $\lambda^{\prime}(K) \neq 0$. Then no surgery in Q- $\{0\}$ on $K$ yields a manifold with cyclic fundamental group.

The argument here indicates a program for proving property $P$. First it should be shown that the fundamental group of a knot admits irreducible representations into $S U(2)$ that map down to immersed arcs and circles in the pillow case. The second thing that needs to be shown is that the arcs and circles of representations move monotonically around the pillow case. Partial results of this nature are also known to $S$. Kerckhoff (unpublished).

\section{REFERENCES}

1. S. Akbulut and J. McCarthy, Notes on Casson's Invariant (Princeton Lecture Notes).

2. M. Culler et al., Dehn surgery on knots, Ann. of Math. 125 (1987), 237-300.

3. C. Frohman, Unitary representations of knot groups, Topology, to appear.

4. C. Frohman and E. Klassen, Deforming representations of knot groups into $S U(2)$, preprint.

5. C. Frohman and D. D. Long, Generalized Casson's invariants, in preparation.

6. C. Frohman and A. Nicas, Knot invariants via intersection homology, in preparation.

7. X.-S. LIN, Representations of knot groups and twisted Alexander polynomials, preprint.

8. A. Lubotzky and A. MAGID, Varieties of group representations of finitely generated groups, Mem. Amer. Math. Soc. 336.

UNIVERSITY OF IOWA, IOWA CiTY, lowa 52242

U.S.A.
UNIVERSITY OF CALIFORNIA, Santa Barbara, CA. 93106 U.S.A. 